\title{
42. Projections from the Anterior Part of the Coronal Gyrus to the Thalamus, the Spinal Trigeminal Complex and the Nucleus of the Solitary Tract in Cats
}

\author{
By Etsuro Kawana and Toshio Kusama \\ Department of Neuroanatomy, Institute of Brain Research, \\ Faculty of Medicine, University of Tokyo \\ (Comm. by Teizo OGAWA, M. J. A., March 12, 1968)
}

In previous studies ${ }^{5), 6)}$ organization of projection fibers of the coronal and sigmoid gyri to the thalamus was investigated. In those studies, however, number of cases with lesions in the anterior part of the coronal gyrus was insufficient, and fibers from the coronal and sigmoid gyri to the ventromedial part of the posteromedial ventral thalamic nucleus (VPM) were not demonstrated. This study has been carried out to observe projection fibers from the anterior part of the coronal gyrus to the thalamus.

Materials and methods. In this study, lesions were made bilaterally in the anterior part of the coronal gyrus in three adult cats $(167,168,172)$, which were sacrificed 7 to 10 days after the operation by an overdose of Nembutal and perfused through the heart with $1.500 \mathrm{ml}$ of $10 \%$ formalin. Exact location of lesions in these cases has been previously reported by Mabuchi and Kusama." The Nauta-Gygax method ${ }^{9)}$ was employed on the serial frozen sections of 25 micra thickness from the brains.

Results and discussion. 1) Projections to the posterior ventral thalamic nucleus (VP). The anterior part of the coronal gyrus sent fibers mainly to the ventromedial part of VPM from 4th to 6th levels (Fig. 1). At the 7th level (Fig. 1) amounts of degenerating fibers in VPM were decreased. The pars medialis of the posterolateral ventral nucleus (VPLm) at the 3rd level received very small number of fibers (Fig. 1). Few if any or no fibers were traced to the pars lateralis of the postero-lateral ventral nucleus (VPLl). Main projectional areas of the anterior part of the coronal gyrus in VPM (Fig. 1) were different from those of its middle and posterior parts, reported in our previous papers (Fig. 2).

The ventromedial part of VPM, which was the thalamic relay nucleus of the tactile sensation from the mouth region, ${ }^{8)}$ received fibers from the anterior part of the coronal gyrus except for its most anterior corner. This cortical area, furthermore, projected 

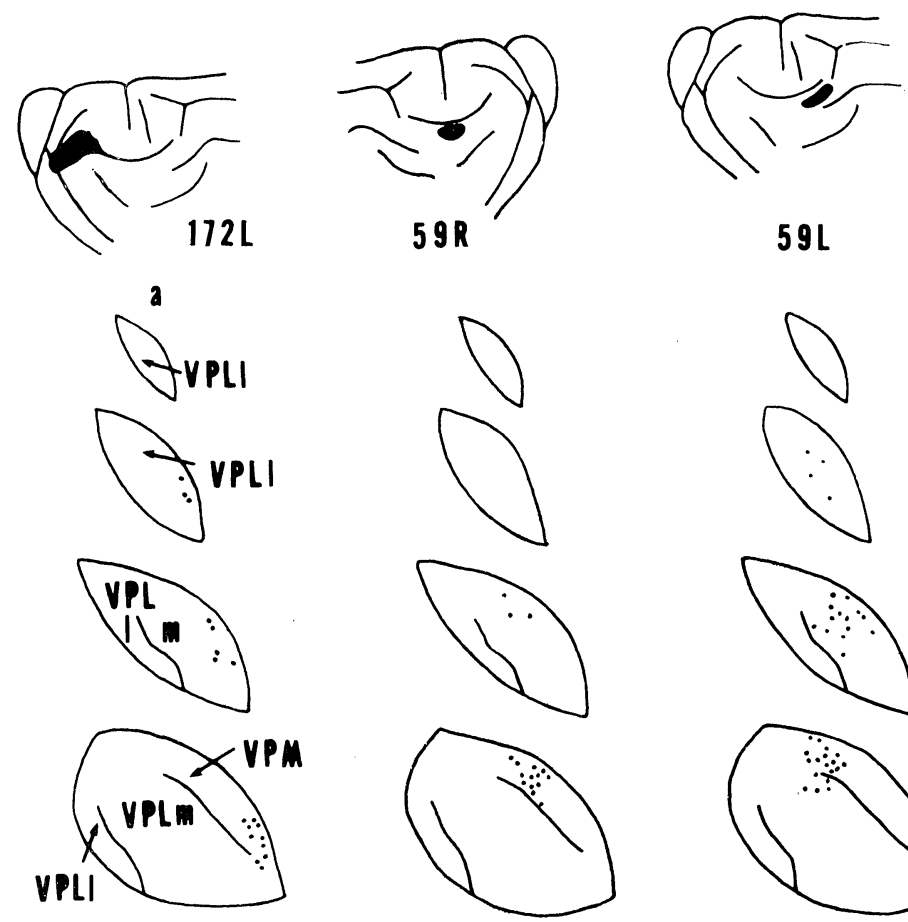

1

2

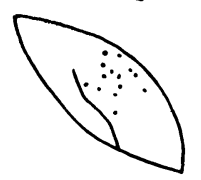

3
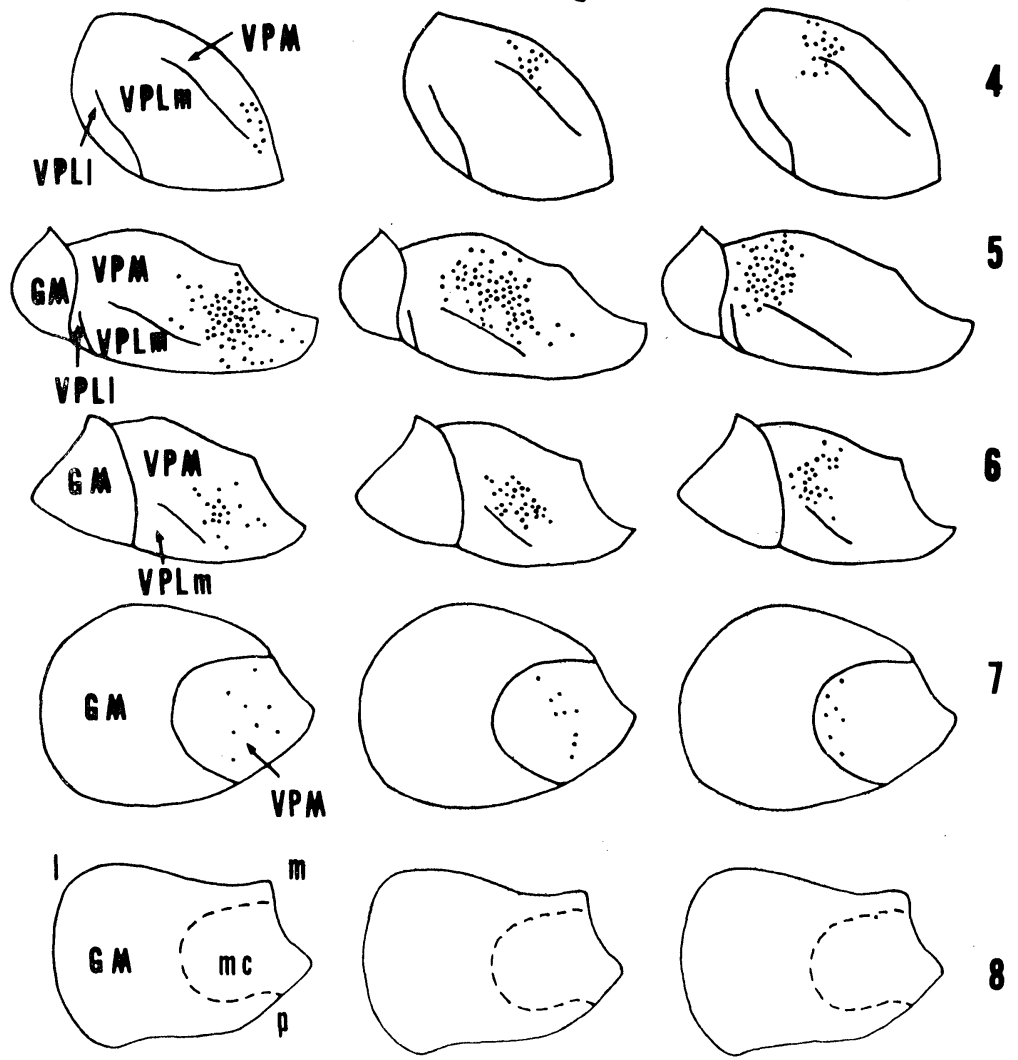

Fig. 1. Diagrammatical representation of degenerating fibers from the anterior part (172L) of the coronal gyrus to the posterior ventral thalamic nucleus in 8 frontal sections numbered from anterior to posterior, comparing with those from the middle (59R) and the posterior parts (59L) of the coronal gyrus, already reported.5),6) Dots showed preterminal degenerating fibers and blackened areas demonstrated lesions in the lateral cerebral cortex. 

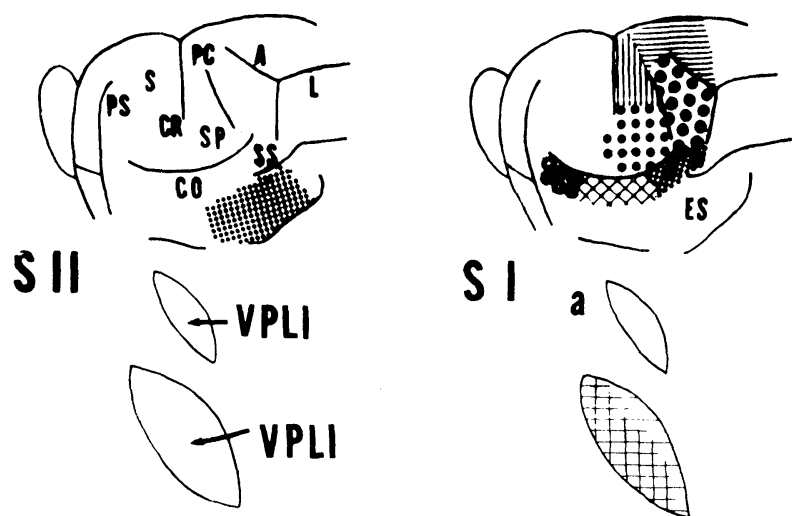

1
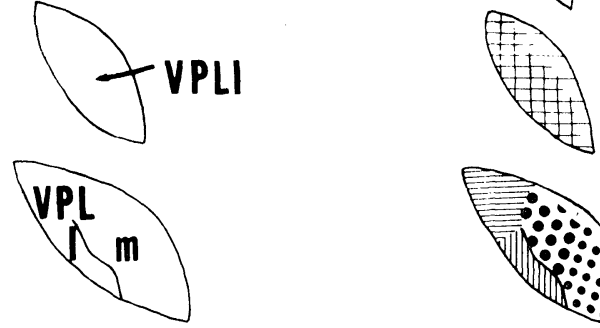

2
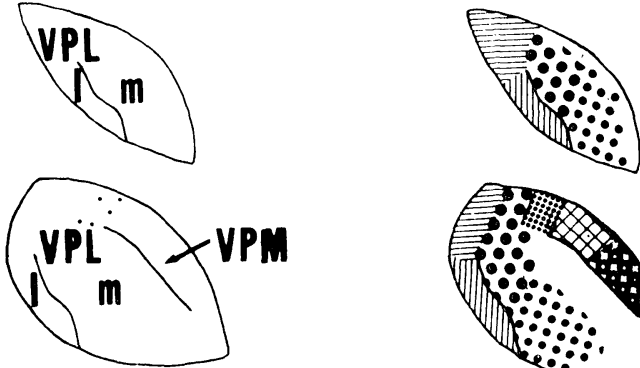

3
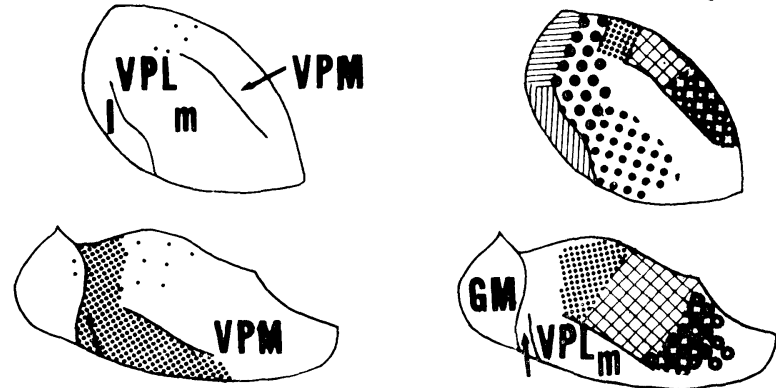

5
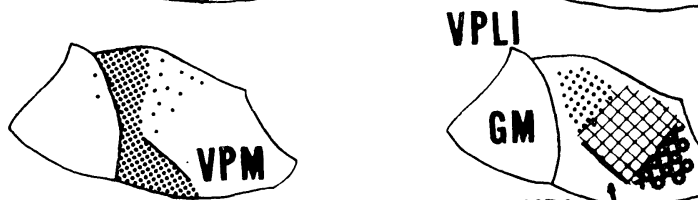

6

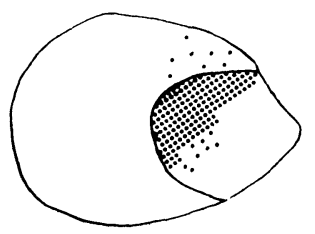

VPLII
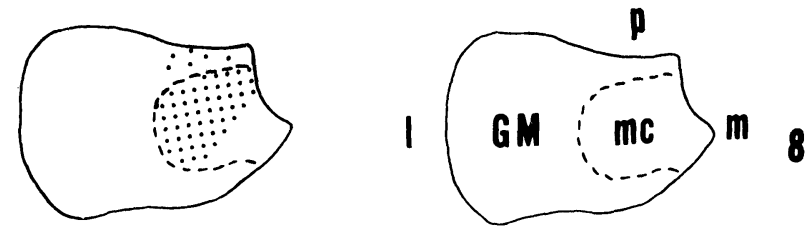

Fig. 2. Diagram showing organization of the corticothalamic (VP) fibers in 8 frontal sections numbered from anterior to posterior in the cat. Corresponding origins and terminations were indicated by the use of identical symbols. 
fibers remarkably to the anterior part of the nucleus of the solitary tract and to the dorsomedial parts of the trigeminal sensory nucleus and of the oral nucleus of the spinal trigeminal complex but very small if any fibers to the caudal nucleus of the spinal trigeminal complex (Fig. 3). Thus our findings suggested that this cortical area was the sensory mouth area, although it was included in the face area of the precentral (MI) motor area by Woolsey. ${ }^{10)}$

Main projection areas of the sigmoid and coronal gyri in VP
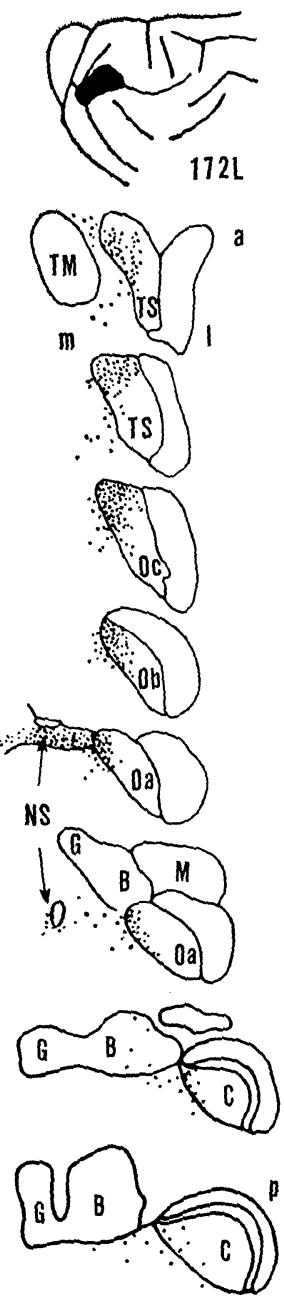
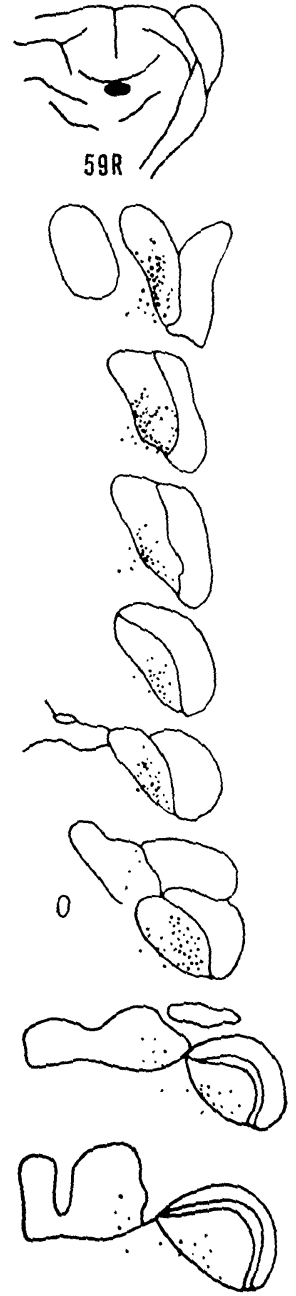
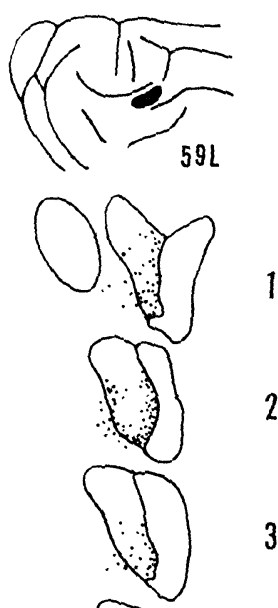

3

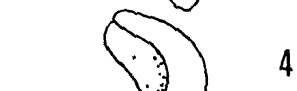

5
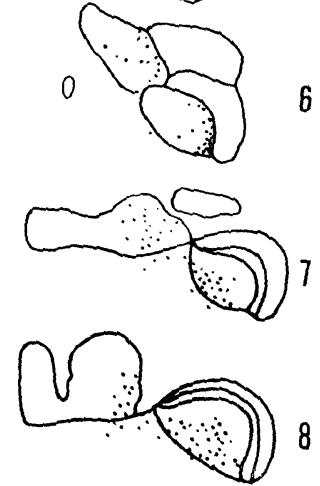

Fig. 3. Diagrammatical representation of degenerating fibers from the anterior part (172L) of the coronal gyrus to the terminal trigeminal nucleus and the dorsal column nucleus in 8 frontal sections numbered from anterior to posterior, comparing with those from the middle (59R) and the posterior parts (59L) of the coronal gyrus, already reported.5),6) Terminology of the terminal trigeminal nucleus described by Eisenman et al. ${ }^{1)}$ was adapted in this study. 
did not extend to the posterior part of VPLl, VPLm, and VPM, as shown in Fig. 2. According to our previous papers, ${ }^{4), 6)}$ these thalamic areas received fibers mostly from the anterior ectosylvian gyrus (the second somatosensory area) (Fig. 2).

2) Projections to the lateral ventral thalamic nucleus (VL) and to the lateral reticular formation of the pons and the medulla oblongata. The area of the coronal gyrus projecting fibers to the medial part of VL and to the lateral part of the reticular formation was much more restricted to its most anterior corner and slightly smaller in size than that described in our previous papers, considering a case (cat no. 172L) with lesions of the anterior part of the coronal gyrus apart from its most anterior corner in which only a small number of fibers were followed to VL and to the lateral part of the reticular formation. Woolsey ${ }^{10)}$ designated the anterior part of the coronal gyrus as the face area of the precentral (MI) motor area. But in our study most of this cortical area sent fibers to the ventromedial part of VPM, mouth sensory area. ${ }^{8)}$ Main projection to the medial part of VL and to the lateral part of the reticular formation of the pons and the medulla oblongata (face motor area) occurred in the most anterior corner of the anterior part of the coronal gyrus and in the anterior part of ASl (the lateral part of the anterior sigmoid gyrus).

According to a cytoarchitectonical study of Gurewitsch and Chatschaturian, ${ }^{2}{ }^{2}$ the anterior part of the coronal gyrus was composed of the area 4. Recently, Hassler and Muhs-Clement ${ }^{3)}$ demonstrated that most of the anterior part of the coronal gyrus was the area $3 \mathrm{a}$ and area 6 was located in its anterior corner. It is very interesting to compare our results with those of Hassler and MuhsClement. ${ }^{3)}$

3) Projections to the other thalamic nuclei.

Projection fibers of the anterior part of the coronal gyrus could be followed to the dorsal medial thalamic nucleus (MD), if the lesions extent into the gyrus proreus. The posterior part of the lateral central nucleus and the nucleus centrum medianum received a small number of fibers from the anterior part of the coronal gyrus. Projection fibers to LP were not demonstrated from this cortical area.

Acknowledgement. The authors wish to express their gratitude to Mrs. K. Toyoshima-Kobayashi for her technical assistance. 


\section{References}

1) Eisenman, J. et al. (1963): Acta physiol. scand., 59, suppl., 214, 1-44.

2) Gurewitsch, M., and Chatschaturian, A. (1928): Z. Anat. Entwickl.-Ges., 87, 100-138.

3) Hassler, R., and Muhs-Clement, K. (1964): J. Hirnforsch., 6, 377-420.

4) Kawana, E. (1966): Acta Anat. Jap., 41(5), suppl. 11 (in Japanese).

5) Kawana, E., and Kusama, T. (1964): Folia Psychiat. Neurol. Jap., 18, 337-380.

6) Kusama, T., et al. (1966): Progress in Brain Research, 21A, 292-322, Elsevier (Amsterdam).

7) Mabuchi, M., and Kusama, T. (1966): Brain Research, 2, 254-273.

8) Mountcastle, V. B., and Henneman, E. (1949): J. Neurophysiol., 12, 85-100.

9) Nauta, W. J. H., and Gygax, P. A. (1954): Stain Techn., 29, 91-93.

10) Woolsey, C. N. (1958): Biological and Biochemical Bases of Behaviour. University of Wisconsin Press, Madison, 63-81. 